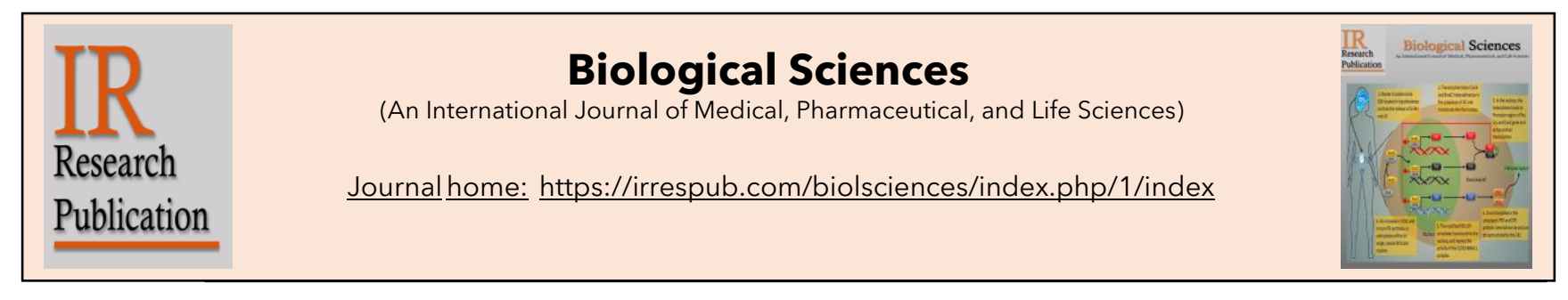

\title{
Development and Evaluation of Resveratrol Loaded Modified Oleic Acid Based Vesicles for the Treatment of UV-B Induced Precancerous Skin Lesions
}

\author{
Arun Singh Lalotra*, Vishesh Singh \\ Department of Pharmaceutics, ISF College of Pharmacy, Moga, Punjab, 142001, India
}

\author{
ARTICLE HISTORY \\ Received: 10-04-2021 \\ Revised: 12-04-2021 \\ Accepted: 12-04-2021 \\ Online: $12-04-2021$

\section{KEYWORDS} \\ Skin cancer \\ Resveratrol \\ Oleic acid vesicles \\ Central composite design \\ Skin permeation studies \\ Skin deposition
}

\section{ABSTRACT}

Skin cancer is associated with the abnormal growth of skin cells; it most often develops on skin exposed to the sun. The conventional treatment such as cream, gel, etc. are less flexible, so they don't penetrate deep into the skin (dermal layer), it remains at the upper part of the skin (epidermal layer). Stratum corneum acts as a physiological barrier for the drug-loaded in cream and gel. An oleic acid-based vesicle is used to deliver the bioactive Resveratrol into the skin to overcome the stratum corneum. The scope of the current study is to develop Resveratrol-loaded oleic acid vesicles, which are used to enhance the permeability of drugs into the deeper layers of the skin and overcoming the stratum corneum barrier. The proposed study claims that it can retain the permeated bioactive into the deeper layer for a longer duration to get the sustained effect of the drug in the dermal region and avoid the systemic exposure and toxicity of the drug. Resveratrolloaded oleic acid vesicles would be a promising approach for the topical delivery of drugs/bioactive for the treatment of UV-Binduced precancerous skin lesions.

\section{Introduction}

Skin is the body's largest organ, covering an

*Address for correspondence

Department of Pharmaceutics,

ISF College of Pharmacy, Moga (Punjab), India

Email: laotraarun899@gmail.com

DOI: http://dx.doi.org/10.55006/biolsciences.2021.1104 Published by IR Research Publication; Lalotra A et al (c) 2021 by Biological Sciences is licensed under CC BY 4.0 (C) impressive surface area of about $2 \mathrm{~m} 2$ and representing about $20 \%$ of total adult weight. It is an important barrier, situated at the interface between the human body and the environment, with both passive and active roles against chemical, physical and microbial insults [1]. Its well-characterized mechanical barrier feature restricts water loss. It prevents the entry of potentially harmful substances and micro-organisms and forms an active barrier that provides the first line of immunological protection against infection [2]. Human skin consists of three different layers: epidermis, dermis, and hypodermis. The epidermis is the most fragile and biologically active of these layers, as the epithelium basal layer (stratum basal) is continuously being renewed. Despite the high turnover on the skin 
surface, a stable landscape of fractal geometric patterns is seen [3]. The epidermis layers include the basal stratum, stratum spinosum, stratum granulosum, stratum lucidum, and stratum corneum. The dermis is between the epidermis and hypodermis and is responsible for regional skin thickness variation. It is primarily composed of collagen but also includes elastin, blood vessels, nerves, and sweat glands. The papillary dermis is situated under the dermal-epidermal junction and comprises a loose mixture of fibrocytes, collagen, and vessels of blood. The much thicker reticular dermis lies under it. It contains fewer fibrocytes but a more robust collagen array [4]. The hypodermis consists mainly of loose connective tissue, which forms gliding layers or large pockets of adipose tissue that insulates and protects the skin depending on the region. The deepest extent of the hypodermis is completely free from fat and is best known for the messy pattern of fibers [3].

The most prevalent type of cancer is skin cancer. This is classified into two sub-categories: nonmelanoma skin cancer (NMSC), and melanoma. NMSC consists of squamous cell carcinoma (SCC) and basal cell carcinoma (BCC). Due to its increasing incidence, NMSC has become a growing public health concern. UV-Radiation (UV-R) is the primary cause of skin cancer since large amounts of UV-R touch the earth's surface [5]. Two forms of UV radiation are primarily responsible for cancerous skin damage: UVA $(315 \mathrm{~nm}-400 \mathrm{~nm})$ and UVB $(280 \mathrm{~nm}-315 \mathrm{~nm})$. In sunlight, UVA is much more abundant than UVB, containing $95 \%$ of solar UV radiation. UVA has a deeper penetration into the dermis than UVB. UVB may penetrate several layers of cells into the skin's dermal layer, perhaps also into the layer of basal cells. The types of UVB induced DNA photodamage include pyrimidine dimer, purine and pyrimidine dimer, purine photoproduct and protein-DNA cross-link, and single-strand break, of which pyrimidine dimer is the most important form of DNA photodamage [6].

Resveratrol (trans-3,5,4-trihydroxystilbene) is a polyphenolic compound that is synthesized to respond to ecological strain and pathogenic infection in grapes, berries, peanuts, and plants [7]. Resveratrol has a wide range of biological effects, including antioxidant, cardioprotective, neuroprotective, anti-inflammatory, anti-cancer, and anti-diabetic activity. In several recent studies, Resveratrol's chemopreventive and therapeutic actions have been well defined [8]. Resveratrol protects against skin cancer mediated by UVB. Resveratrol significantly inhibits the induction of multiple UVB-mediated epidermal hyperplasia by decreasing the proliferation of nuclear cell antigen and decreasing the regulation of CDK-2, -4 , and -6 , as well as cyclin-D1 and -D2. Additionally, Resveratrol prevents skin photodamage by inducing p66Shc phosphorylation in $\mathrm{HaCaT}$ cells[8]. Oleic acid vesicles (OAVs) have also been previously documented to enhance the absorption of drugs through the skin. OAVs were used via topical administration as the carrier for a variety of drugs in studies [11].

\section{Materials and methods}

\section{Materials}

Pure resveratrol sample was collected ex-gratis from Sami labs, Bangalore, India. Phospholipid i.e., Soya phosphatidylcholine (PC) (99\% pure), carbopol 934P, was purchased from Sigma Aldrich, USA. Tween 80, chloroform, methanol, glycerol, and other solvents were obtained from Merck Ltd., India. All other chemicals and reagents were collected from a local supplier and of analytical grade.

Formulation of Resveratrol loaded oleic acid vesicles

Resveratrol-loaded modified oleic acid vesicles were prepared by using soya lecithin ( $88 \mathrm{mg}$ ), tween80 (60 mg), oleic acid (50 mg), and bioactive $(10 \mathrm{mg}$ ) in RBF, then methanol and chloroform were added to dissolve all the ingredients completely. The RBF is then placed in a rotary evaporator and makes the thin film by using Rota-Evaporator at temperatures $350 \mathrm{C}$ to $400 \mathrm{C}$. Resveratrol-loaded oleic acid-based vesicles are obtained after hydration with glycerol solution $(1.5 \mathrm{~mL}$ glycerol and $8.5 \mathrm{~mL}$ water).

\section{DoE based optimization by applying Central Composite Design}

Optimization was done to get the desired vesicle size, zeta potential, and maximum entrapment efficiency. The following formulation and independent variables were optimized:

$\mathrm{X} 1$ = Amount of soya lecithin

X2 $=$ Weight of Tween-80

The Face Centered Central Composite Design was implemented for the optimization of the amount of soya lecithin (X1), and weight of Tween-80 (X2) was independent variables and vesicles size (R1), Polydispersity index (R2), zeta potential (R3), and 
Entrapment efficiency (R4) were selected as a response or dependent variables. High and low levels of each factor were coded +1 and -1 , respectively, as shown in Table $\mathbf{1}$. According to the design, 13 formulations were prepared and evaluated for response. All the batches were evaluated for vesicle size, Polydispersity index, zeta potential, and entrapment efficiency by using the design of expert 12.0.7.0, Stat-Ease.

Table 1. Experimental FCCCD design used for the optimization

\begin{tabular}{|l|l|l|}
\hline Coded levels & Actual values & Actual values \\
\cline { 2 - 3 } & $\mathrm{X} 1(\mathrm{mg})$ & $\mathrm{X} 2(\mathrm{mg})$ \\
\hline-1 & 60 & 20 \\
\hline 0 & 80 & 40 \\
\hline+1 & 100 & 60 \\
\hline
\end{tabular}

Response surface plot

Contour plot and 3D response surface plots were constructed to establish an understanding of the relationship between variables and their interaction.

\section{Optimization using desirability function}

The formulations were optimized by keeping the $X 1$ and $\mathrm{X} 2$ within the range used in the present work while R1 was considered a minimum, R2 and R3 were in range, but $\mathrm{R} 4$ was considered maximum using Design-Expert software. Based on these assigned goals, the software has determined the possible formulation composition with maximum desirability value.

\section{Checkpoint analysis}

According to the desirability value and composition of variables, the final optimized formulation was prepared and evaluated for response. The predicated and observed response was compared, and the percentage prediction error was calculated to confirm the validity of the design for optimization. According to the design, 13 formulations were prepared and evaluated for response. All the experimental batches were evaluated for particles for vesicle size, polydispersity index, and zeta potential. The following factorial batches were prepared (Table 2).

Table 2. Factorial batches for optimization

\begin{tabular}{|l|l|l|}
\hline & Factor 1 & Factor 2 \\
\hline
\end{tabular}

\begin{tabular}{|l|l|l|l|}
\hline Std & Run & $\begin{array}{l}\text { A: Amount of soya } \\
\text { lecithin } \\
\text { (mg) }\end{array}$ & $\begin{array}{l}\text { B: weight of } \\
\text { Tween-80 } \\
\text { (mg) }\end{array}$ \\
\hline 12 & 1 & 80 & 40 \\
\hline 9 & 2 & 80 & 40 \\
\hline 10 & 3 & 80 & 40 \\
\hline 5 & 4 & 60 & 40 \\
\hline 13 & 5 & 80 & 40 \\
\hline 3 & 6 & 60 & 60 \\
\hline 1 & 7 & 60 & 20 \\
\hline 2 & 8 & 100 & 20 \\
\hline 6 & 9 & 100 & 40 \\
\hline 8 & 10 & 80 & 60 \\
\hline 7 & 11 & 80 & 20 \\
\hline 4 & 12 & 100 & 60 \\
\hline 11 & 13 & 80 & 40 \\
\hline
\end{tabular}

Formulation of Resveratrol oleic acid vesicles carbopol hydrogel

$750 \mathrm{mg}$ carbopol 934P was dispersed in $100 \mathrm{~mL}$ of warm distilled water and stirred at 250-350 rpm until polymer was dispersed homogeneously and then neutralized using 4-5 drops of triethanolamin. It was homogenized until it became a transparent gel. The optimized formulation of oleic acid vesicles was then gently levigated to formulate the Resveratrol loaded modified oleic acid vesicles carbopol hydrogel in the developed carbopol gel base in the ratio of 5:1. Also prepared for comparative studies containing the same amount of Resveratrol was conventional plain resveratrol carbopol hydrogel (Res-CBP-Gel). Similarly, this resveratrol solution in the developed carbopol gel base was prepared and levigated. The gel was visually evaluated for its color and physical consistency as well as other physical and chemical properties [14].

\section{Formulation of conventional cream of Resveratrol}

In different comparisons Resveratrol paid conventional cream (Res-Cream) was also developed. This consisted of 3\% resveratrol, $6 \%$ span $80,3 \%$ white beeswax, 36\% white soft paraffin, $15 \%$ liquid paraffin, and sufficient distilled water. To formulate the cream, a simple emulsification method was employed to melting and mixing the components with stirring [15].

\section{Evaluation of physicochemical characteristics}


Transmission electron microscope (H 7500 Hitachi, Japan) was used for morphology (i.e., uniformity of shape and lamallarity). Prepared formulaions were 100 times diluted with triple distilled water and negatively stained with $s$ solution of phosphotungstic acid ( $1 \% \mathrm{w} / \mathrm{v})$. Images were taken with microscopy [16].

\section{Micromeritic studies}

Zeta sizer (Malvern Instruments, UK) was used to determine vesicle size, size distribution, and zeta potential of vesicles and vesicles loaded hydrogel. The formulation was diluted five times with normal saline $(0.9 \% \mathrm{w} / \mathrm{v})$. Microscopically, hemocytometer was used to count the number of vesicles per cubic $\mathrm{nm}$. The number of vesicles was counted in 80 small squares, and the average number of vesicles was calculated using the following formula [15].

\section{Total no of vesicles \\ $=($ Total no of vesicle counted in 80 small squares \\ $\div$ Total number of squares counted) \\ $\times$ Dilution factor}

Drug entrapment efficiency \% (EE\%)

The entrapment efficiency of RES in oleic acid vesicles was calculated from micellar dispersion obtained by filtration via Sephadex G-50 column after separation of the unincorporated drug while centrifugation for 3 minutes at 3000 RPM. EE\% of RES was spectrophotometrically analyzed at $\lambda$ max 306 nm (Shimadzu UV-1601 PC, Kyoto, Japan) after sufficient water dilution. RES concentration was determined using the solution of $4 \mathrm{~mL} \mathrm{NaCl}(0.9 \%)$, $1 \mathrm{~mL}$ Triton X-100 (0.5\%) as blank for baseline correction, and then the concentration of obtained micelles dispersion was determined. Each experiment was carried out in triplicate, and the mean value was deduced as per the formula.

$\mathrm{EE} \%=$ Concentration $\times$ Dilution factor $\times$ Volume

Viscosity, spreadability, and $\mathrm{pH}$ determination

The Resveratrol loaded modified oleic acid vesicles hydrogel viscosity was determined using Brookfield R/S plus viscometer (Brook-field Engineering Laboratories, Inc., Middleboro, MA). The spindle R3C75 was used, and the speed was adjusted at 100 RPM. A small amount of the formulations was added at a temperature of $25 \pm 2^{\circ} \mathrm{C}$ at the base of the viscometer. A shear rate range was applied from 1 to $100 \mathrm{~s}-1$ [17]. The spreadability reflects the index of ease of application of topical skin formulation. The plate method was used to calculate the spreadability of gel samples [18]. Two acrylic plates were used, and carbopol gel incorporated in 500 $\mathrm{mg}$ of vesicles dispersion was placed in the middle of the one plate and another placed above it. The initial diameter was determined of the circle in which the gel was distributed. A weight of $500 \mathrm{gm}$ was then placed on the upper plate for $5 \mathrm{~min}$. The spreading of gel as a function of the applied weight was determined by observing the increase in diameter and measurement of the spreadability. A calibrated $\mathrm{pH}$ meter (AE Max) was also used to calculate the $\mathrm{pH}$ of the prepared formulations.

\section{Ex vivo skin permeation studies}

Ex vivo skin permeation was performed using freshly excised pig ear skin (obtained from a local slaughterhouse). The skin of pig was used because it is similar to human skin in the histological context. The stratum corneum of pig pinna, which has a thickness of 21-26 $\mu \mathrm{m}$ and an average of 20 hair follicles per centimeter square, is very similar to the skin of the human forehead, with 14-32 hair follicles per centimeter square. Besides similarity, its simple accessibility and wide use in skin permeation studies make it the preferable model to use in this study [19]. Shaved dorsal skin portion was placed on a static Franz diffusion cell with $3.14 \mathrm{~cm} 2$ crosssectional area and $30 \mathrm{~mL}$ capacity. In the receptor compartment, a mixture of ethanol and buffer $\mathrm{pH}$ 7.4 in a ratio of $3: 7(\mathrm{v} / \mathrm{v})$ was taken, maintained at $32 \pm 0.5^{\circ} \mathrm{C}$ at $100 \mathrm{RPM}$. The donor compartment was filled with different formulations equal to $1.5 \mathrm{mg}$ of the drug (vesicle dispersions, oleic acid vesicles carbopol hydrogel, ethanolic solution, conventional plain Resveratrol containing carbopol hydrogel (Res-CBP-Gel), and conventional Resveratrol (Rescream). At the fixed time $(1,2,4,6,8,10,12,18$, and $24 \mathrm{~h}), 0.5 \mathrm{~mL}$ samples were extracted from the receptor compartment accompanied by fresh media replenishment and spectrophotometrically analysis of drug concentration in the sample. Cumulative amounts of the permeated drug (Qn) were determined using the following equation.

$$
\mathrm{Qn}=\mathrm{Cn} * \mathrm{Vo} \sum_{i=1}^{n=1} \mathrm{Ci} * \mathrm{Vi}
$$

Where, at any time of sampling, Cn represents the drug concentration of the receptor compartment, $\mathrm{Ci}$ is the drug concentration of the ith sample and VO and $\mathrm{Vi}$ is the volumes of the receptor compartment and the sample withdrawn. Permeation curves were 
constructed by plotting against time the cumulative volume of drug that was permeated across the unit area of the skin. The drug's permeation rate (flux; J) was determined from the slope of the gression lines, fitting the linear portion of the permeability profiles [12].Whereas, the coefficient of permeability (Kp) was determined using the following equation.

$$
K p=\frac{J s s}{C o}
$$

Where, the Co is the initial concentration of drugs in the donor cell. Enhancement ration (Er) was determined by dividing Jss of control formula (conventional cream) from the respective formulation by Jss.

\section{Skin deposition studies}

The amount of drug deposited in the dermal layer of the skin after studies of permeation was also analyzed by the method as previously reported [12]. The permeation studies were stopped after $24 \mathrm{~h}$, and the skin was very carefully removed from the diffusion cell. The remaining skin formulation was removed, and the skin was washed and cleansed with triple distilled water and dried with a hairdryer. The skin was cut into small pieces, and the drug was extracted with methanol for $1 \mathrm{~h}$ using bath sonicator sonication. The resulting solution was filtered through a membrane $(0.45 \mathrm{~mm})$, and the filtrate was analyzed for the amount of drug deposited in the skin area (SD, $\mu \mathrm{g} \mathrm{cm}-2$ ) per unit.

\section{Statistical analysis}

The statistical evaluation of the results was carried out using the software Graph Pad Instat 3. All values are expressed as mean \pm S.D. Student t-test has been used to compare mean values of various groups. Statistical significance is $p<0.05$. Multiple comparisons were made using one-way analysis of variance (ANOVA) accompanied by post hoc analysis using the Tukey method. Statistical relevance was considered.

\section{Results and discussion}

Formulation and optimization of Resveratrol loaded modified oleic acid vesicles

The formulation was prepared by the thin-film method. DoE based optimization by applying Central Composite Design was done. Optimization of Resveratrol loaded modified oleic acid vesicles was done to get desired vesicles Size, polydispersity index, zeta potential, and maximum entrapment efficiency. The formulation parameters optimized as shown in Table 3.

Table 3. Variables in FCCD for the formulation

\begin{tabular}{|c|c|c|c|c|}
\hline \multirow[t]{2}{*}{$\begin{array}{l}\text { Independent } \\
\text { Factors (CMA) }\end{array}$} & \multirow[t]{2}{*}{ Units } & \multicolumn{3}{|c|}{$\begin{array}{l}\text { The level used actual } \\
\text { (Coded) }\end{array}$} \\
\hline & & $\begin{array}{l}\text { Low } \\
(-1)\end{array}$ & $\begin{array}{l}\text { Medium } \\
(0)\end{array}$ & $\begin{array}{l}\text { High } \\
(+1)\end{array}$ \\
\hline $\begin{array}{l}\text { Amount of soya } \\
\text { lecithin }\end{array}$ & $\mathrm{mg}$ & 60 & 80 & 100 \\
\hline weight of tween 80 & $\mathrm{mg}$ & 20 & 40 & 60 \\
\hline $\begin{array}{l}\text { Dependent } \\
\text { variables (CQA) }\end{array}$ & Units & \multicolumn{3}{|c|}{\begin{tabular}{|l} 
Constraints \\
\end{tabular}} \\
\hline Vesicles size & $\mathrm{nm}$ & \multicolumn{3}{|c|}{ Range $(100-500 \mathrm{~nm})$} \\
\hline $\begin{array}{l}\text { Polydispersity } \\
\text { Index }\end{array}$ & - & \multicolumn{3}{|c|}{ Minimum } \\
\hline Zeta potential & $\mathrm{mV}$ & \multicolumn{3}{|c|}{ Within range } \\
\hline $\begin{array}{l}\text { Entrapment } \\
\text { Efficiency (EE) }\end{array}$ & $\%$ & \multicolumn{3}{|c|}{ Maximum } \\
\hline
\end{tabular}

Quantitative aspects of the effects and relationships among various formulation variables of RES loaded oleic acid vesicles were investigated using Response Surface Methodology (RSM). FCCC Design with a total of 13 experimental runs was selected to optimize the various process parameters at three levels and evaluated for response. Optimization of the variable using design-Expert software (version 12.0.7.0, Stat-Ease, Inc.) shown in Table 4.

Fitting the model to data

Table 5 shows the results of dependent variables, mean vesicle size (R1), polydispersity index (R2), zeta potential (R3), and entrapment efficiency (R4) of the formulations. All data were generated by Design-Expert software in which every response was fitted to a second-order quadratic model, and model significance was identified by ANOVA, lackof-fit test, and multiple correlation coefficient tests. In the ANOVA test, the model p-values for R1, R2,

Table 4. Optimization by Face Cantered Central Composite Des

\begin{tabular}{|l|l|l|l|l|l|}
\hline & & Factor 1 & Factor 2 & Response 1 & Res \\
\hline Std & Run & $\begin{array}{l}\text { A: Amount. of } \\
\text { soya lecithin }\end{array}$ & $\begin{array}{l}\text { B: Weight of } \\
\text { Tween-80 }\end{array}$ & Vesicle Size & PDI \\
\hline ublication - All R & ghts Reserved & $\mathrm{mg}$ & $\mathrm{nm}$ & \\
\hline 12 & 1 & 80 & 40 & $403.7 \pm 31.45$ & 0.21 \\
\hline 0 & 2 & 00 & 10 & $1001 \pm 0007$ & 010
\end{tabular}


R3, and R4 are less than 0.05, so all responses fitted the quadratic model. The lack-of-fit test describes the difference of the data around the fitted model. A model with a significant lack-of-fit lacks prediction efficiency. In Table 2, the coefficients (factor intercepts) with more than one term $(X 1 * X 2)$ and those with high-order terms (X1 and $X 2$ ) indicate the interaction and quadratic effects, respectively.

Influence of formulation variables on the response (CQAs)

Different factors amount of soya lecithin (X1) and weight of Tween-80 (X2) affecting the different critical quality attributes of formulation, i.e., vesicle size (Y1), Polydispersity index (Y3), Zeta potential $(Y 3)$, and Entrapment efficiency (Y4). The influence of variables on critical quality attributes are shown in

Figures 1, 2, and $\mathbf{3}$ for vesicle size, for polydispersity index are shown in Figures 4, 5, and $\mathbf{6}$ for zeta potential effects are shown in Figures 7, 8, and 9. The effect of factors on entrapment efficiency is shown in Figures 10, 11, and 12.

\section{Response R1 Vesicles size}

With an increase in the concentration of $A$ the vesicles size increase and with an increase in the concentration of $B$ the vesicles size decrease. In
Table 5. Summary of results of regression analysis for responses R1, R2 and R3 for fitting to following models

\begin{tabular}{|l|l|l|l|l|}
\hline Model & Response & $\mathrm{R}^{2}$ & $\begin{array}{l}\text { Adjuste- } \\
\mathrm{d} \mathrm{R} \mathrm{R}^{2}\end{array}$ & $\begin{array}{l}\text { Predicte- } \\
\mathrm{d} \mathrm{R}\end{array}$ \\
\hline Linear & $\mathrm{R} 1$ & 0.9275 & 0.9380 & 0.8818 \\
\hline Linear & $\mathrm{R} 2$ & 0.8966 & 0.8809 & 0.9070 \\
\hline Quadratic & $\mathrm{R} 3$ & 0.9315 & 0.9276 & 0.8865 \\
\hline Quadratic & $\mathrm{R} 4$ & 0.9478 & 0.9697 & 0.8923 \\
\hline
\end{tabular}

Figure 1, perturbation curves clearly show the positive effect of $X 1$ and the negative effect of $X 2$. Contour plot and the 3D response of vesicles size is shown in Figure 2 and $\mathbf{3}$.

Response R2 Polydispersity index

Polydispersity index increases with an increase in the concentration of $A$, and with an increase in concentration of $\mathrm{B}$, the polydispersity index decreases. Perturbation curves clearly show the positive effect of $X 1$ and the negative effect of $X 2$ in

Figure 4. The contour plot and $3 \mathrm{D}$ response are shown in Figure $\mathbf{5}$ and $\mathbf{6}$. 


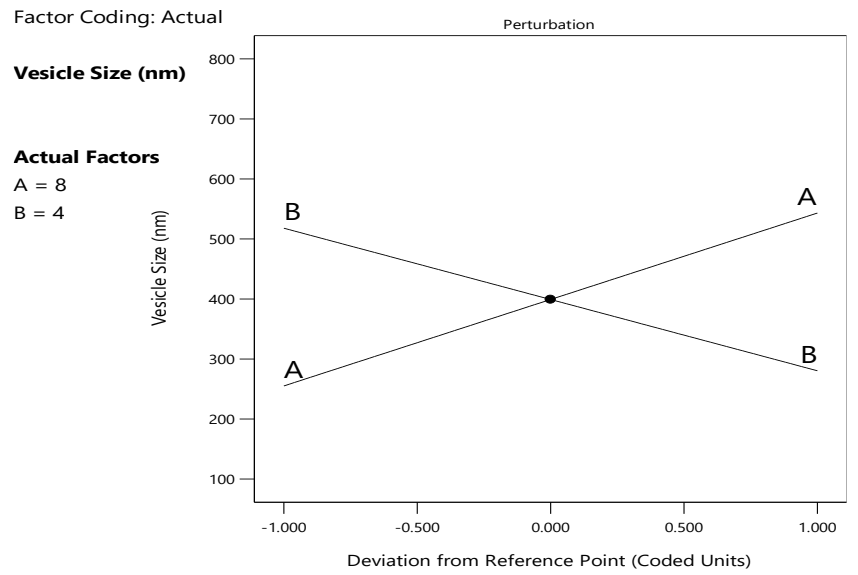

Figure 1. Perturbation and interaction curve showing the effect of factor $A$ and $B$ on vesicles size

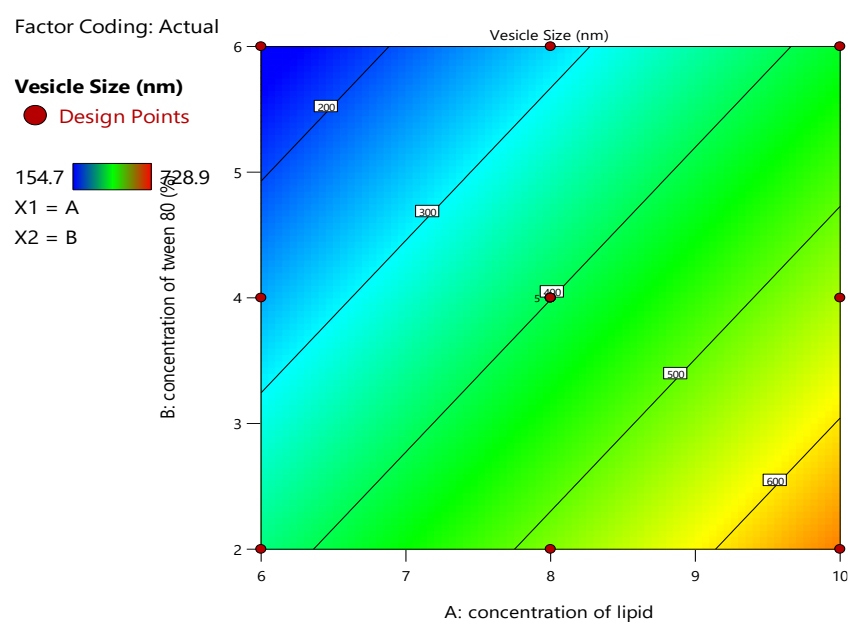

Figure 2. Contour plots showing the showing effect of factor $A$ and $B$ on vesicles size

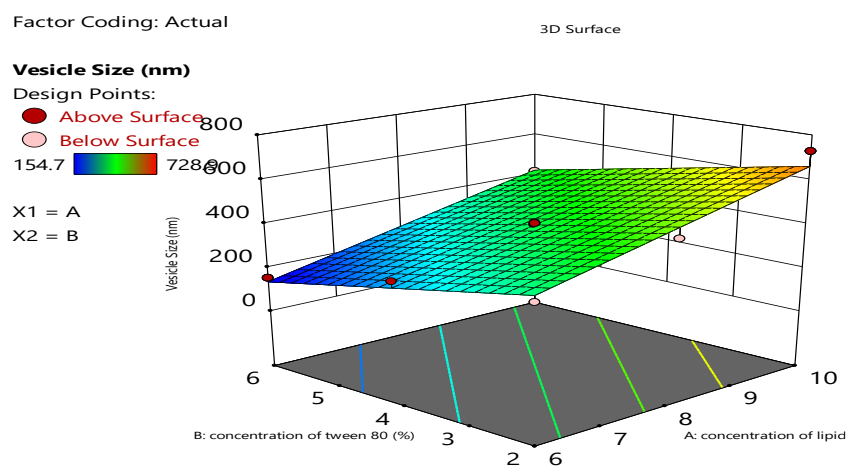

Figure 3. 3D response surface graphs showing the effect of factor $A$ and $B$ on vesicles size

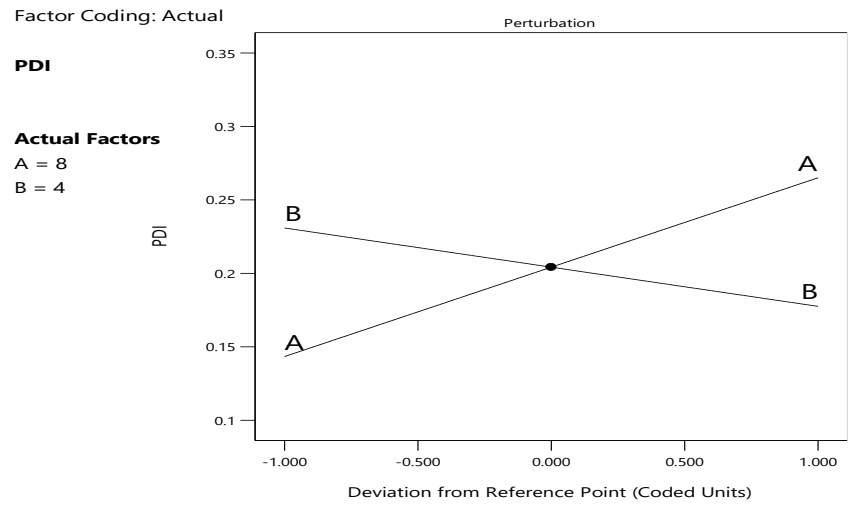

Figure 4. Perturbation and interaction curve showing the effect of factors $A$ and $B$ on the polydispersity index

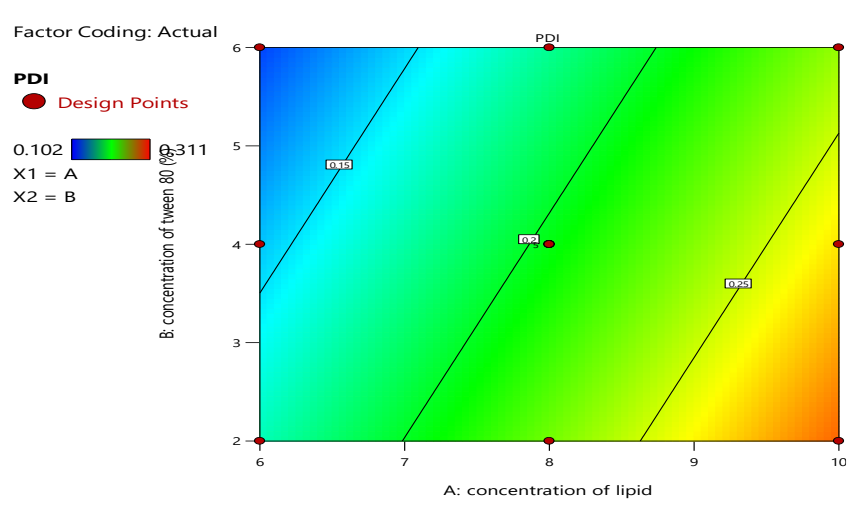

Figure 5. Contour plots showing the effect of factor $A$ and B on polydispersity index

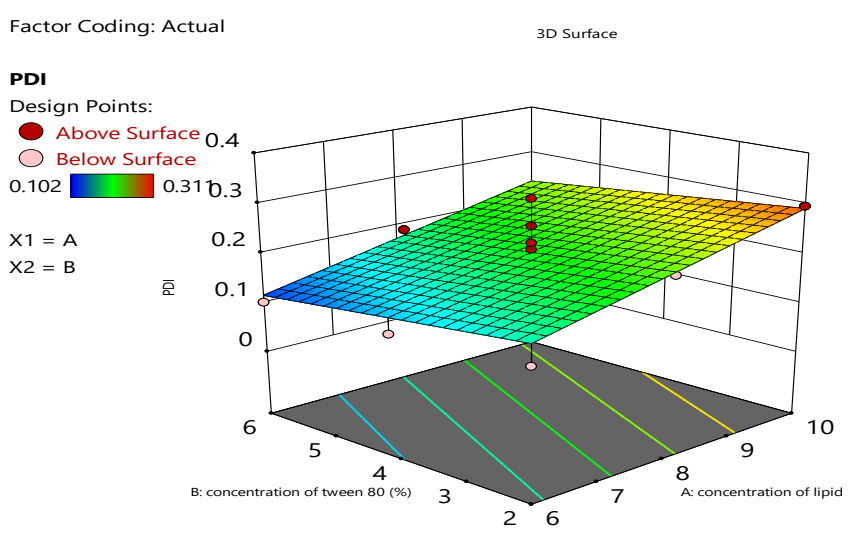

Figure 6. 3D response surface graphs showing the effect of factor $A$ and $B$ on the polydispersity index

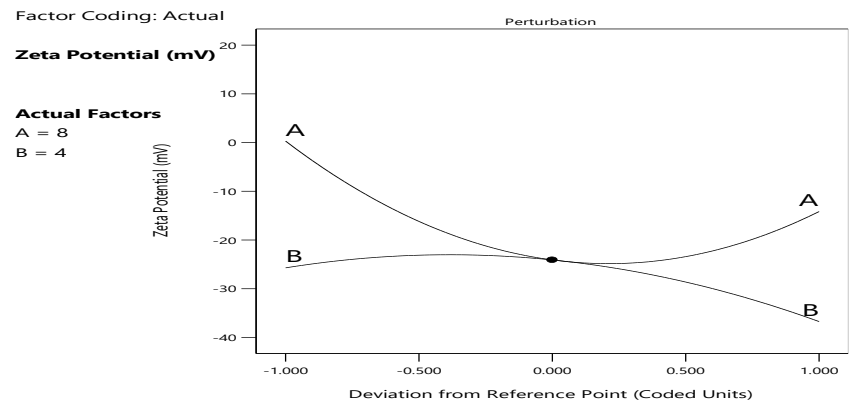


Figure 7. Perturbation and interaction curve showing the effect of factor $A$ and $B$ on zeta potential

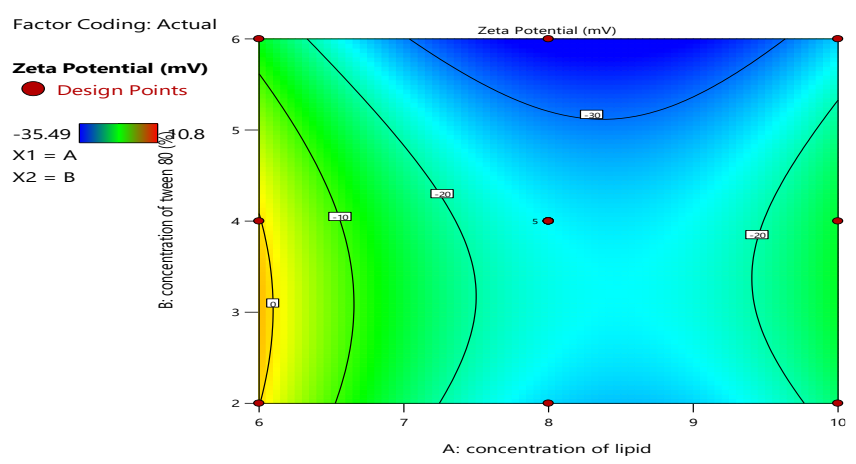

Figure 8. Contour plots showing the showing effect of factor A and B on zeta potential

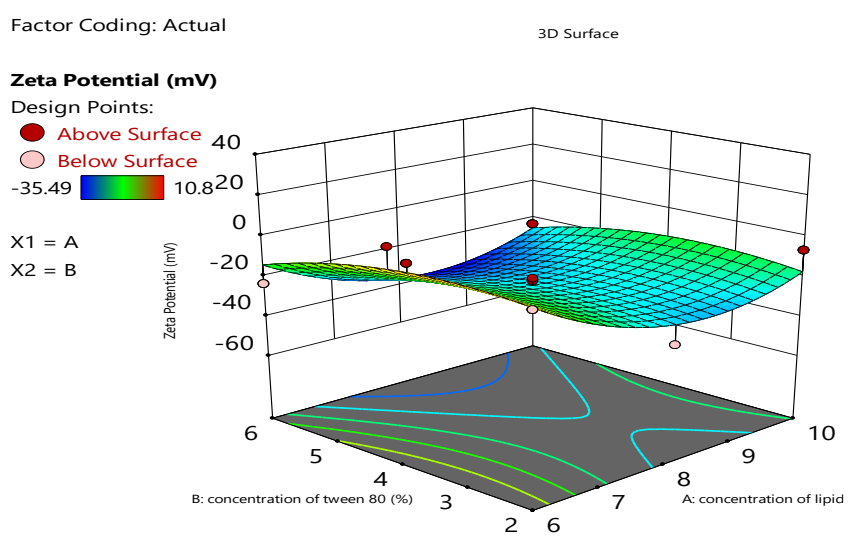

Figure 9. 3D response surface graphs showing the effect of factor $A$ and $B$ on zeta potential

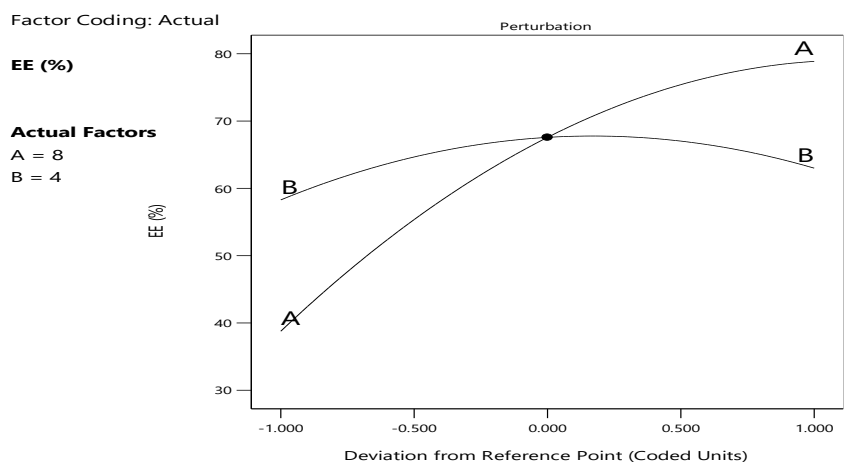

Figure 10. Perturbation and interaction curve showing the effect of factor $A$ and $B$ on entrapment efficiency

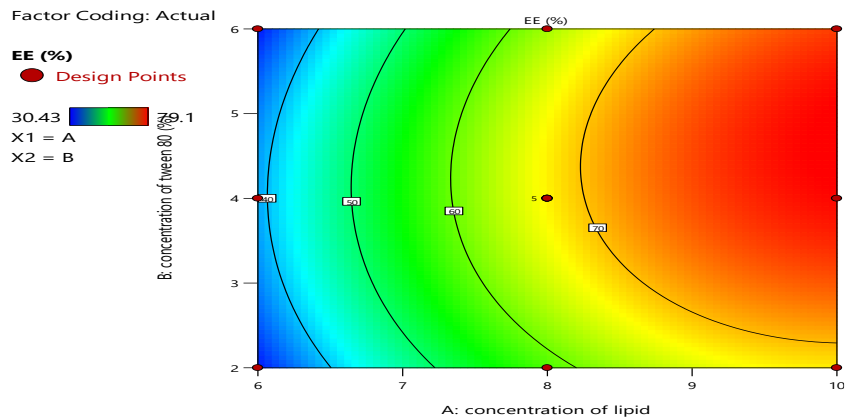

Figure 11. Contour plots showing the showing effect of factor $A$ and $B$ on entrapment efficiency

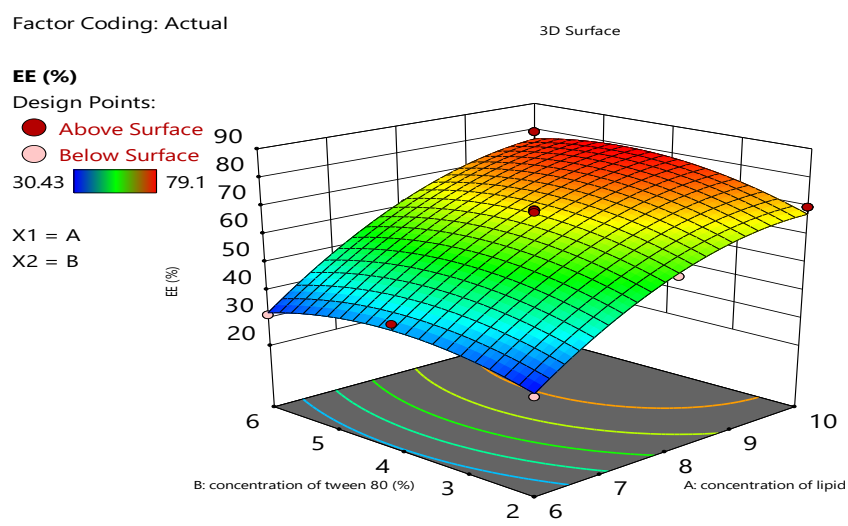

Figure 12. 3D response surface graphs showing the effect of factor $A$ and $B$ on entrapment efficiency

\section{Response R3 Zeta potential}

With increasing in the concentration of $A$ the zeta potential gradually decreases, and with an increase in the concentration of $B$, the zeta potential increases and then decreases after some amount is added, as shown in Figure 7. The contour plot and $3 \mathrm{D}$ response of zeta potential is shown in Figure 8 and 9.

\section{Response R4 Entrapment efficiency}

The entrapment efficiency increases with an increase in the concentration of $A$, and with an increase in the concentration of $B$ the entrapment efficiency gradually increases a little, then decreasing as shown in Figure 10, 11, and 12 shows contour plot and 3D response of entrapment efficiency.

\section{Checkpoint analysis}

A checkpoint analysis was performed to confirm the prediction. There was an excellent agreement between the measured responses and predicted 
responses. The experimental values were very close to the predicted values, with low percentage bias, suggesting that the mathematical model was reliable, and hence, the proposed model can be used to navigate the design space as shown in

\section{Table 6.}

Evaluation of physicochemical characteristics of optimized vesicles formulation and corresponding hydrogel

The average optimized formulating vesicle size was found to be $323.48 \pm 3.06 \mathrm{~nm}$. The PDI value was found to be $0.185 \pm 0.011$, which suggests a homogeneous population. Zeta potential for the optimized formulation of the vesicles was found to be $-34.21 \pm 1.22 \mathrm{mv}$. It was incorporated into the Carbopol hydrogel system due to low viscosity and poor skin applicability of the dispersion of vesicles. This optimized dispersion of the vesicles was integrated into the gel, resulting in improved viscosity. It is useful for better and smoother application of the skin and also important to ensure the consistency and retention of formulation with the gel matrix. Resveratrol-coated hydrogel vesicles with oleic acid were found to be physically stable, free from gritty, and translucent. Microscopic tests have confirmed the integrity of the incorporated vesicles. No evidence of vesicle disruption was found, suggesting that the vesicles were well tolerated for the vigor involved in a hydrogel formulation. The optical microscope image of the optimized formulation of the hydrogel vesicles is shown in Figure 13, indicating that the vesicles have a well-defined structure and spherical shape. No drug loss was observed during the gelling cycle as the drug content was found to be approx. $100 \%$ in Resveratrol loaded oleic acid vesicles. Apparent hydrogel vesicles viscosity was $35.43 \pm 3.22$ Pa.s. Rheological studies have clearly demonstrated the shear-thinning nature of the structure. Viscosity and yield values of the gel were found within the limits of higher plasticity and lower fluidity; hence adequate rigidity of the gel structure is required. Due to its increased viscosity, the spreadability of the hydrogel vesicles $(6.52 \pm 0.39 \mathrm{~cm} 2)$ was slightly less than that of the simple Carbopol hydrogel $(7.02 \pm 0.23 \mathrm{~cm} 2)$. The $\mathrm{pH}$ was found to be $7.6 \pm 0.4$, which shows that there is no risk of causing skin

irritation. The entrapment efficiency was observed $73.2 \pm 0.48$. The formulation was thus considered consistent with the patient and skin-friendly as required with any topical formulation.

\section{Ex vivo skin permeation studies}

The analysis of ex vivo skin permeation was carried out using the freshly excised skin from the pig ear (obtained from the local slaughterhouse). Permeation profiles of vesicular dispersion and vesicular hydrogel were compared with conventional hydrogel and permeation flux values, permeability coefficient, enhancement ratio, and skin deposition, as shown in Table 7. Vesicular dispersion and vesicular hydrogel effectively improved resveratrol permeation compared to plain carbopol gel, showing the enhancement ratio of 2.921 and 2.682 reflected by a high permeation flux i.e., $7.34 \pm 0.61 \mu \mathrm{g} \mathrm{cm}-2 \mathrm{~h}-1$ and $6.94 \pm 0.95 \mu \mathrm{g}$ cm-2 h-1 compared to plain carbopol Gel i.e., $2.66 \pm 0.59 \mu \mathrm{g} \mathrm{cm}-2 \mathrm{~h}-1$ and a statistically significant increase was found $(p<0.01)$. Vesicles cause changes in the lipids; thus, functioning as penetration enhancers are one of the attributed explanations for the results obtained, which many scientists have recently suggested. Another potential issue for improved skin distribution may be the small size of vesicles which provides a higher skin contact surface. The difference between the permeation flux of vesicular dispersion and vesicular hydrogel in vesicles was found to be statistically insignificant ( $p>0.05$ ). It may be attributed to a hydrogel structural 3D network and the carbopol composite structure. 


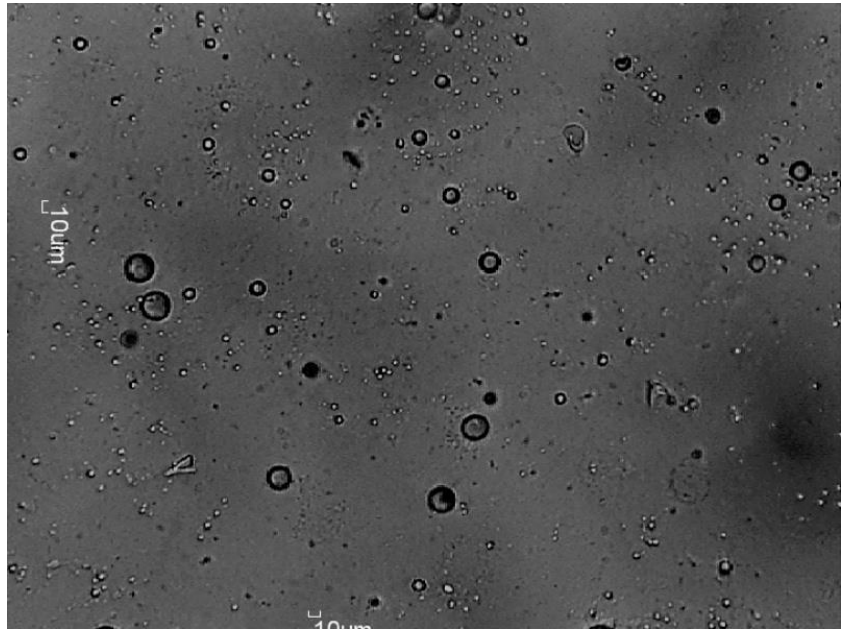

Figure 13. The optical microscope image of the optimized formulation of the hydrogel vesicles

Skin deposition studies

Skin deposition values are represented in table 7. permeation and skin deposition ability compared to traditional and could be used effectively for the topical delivery of Resveratrol.

\section{Conclusion}

Oleic acid vesicles have higher flexibility and have the potential to permeate into a deeper layer of the skin. Oleic acid vesicles enhance the permeability of bioactive Resveratrol into the deeper layers of the skin and overcome the stratum corneum barrier and retain the permeated bioactive into the deeper layer for a longer duration and get the sustained effect of the drug in the dermal region and avoid the systemic exposure of the encapsulated bioactive. desirable dermatological properties of the topical formulation. It was found that the Resveratrol loaded modified oleic acid vesicular hydrogel is stable and efficiency of vesicles. Supported by regression analysis, it has been confirmed that formulation critical material attributes that significantly influence the vesicle size, PDI, Zeta potential, and entrapment.

Table 7. Permeation parameters of different Resveratrol formulations

\begin{tabular}{|l|l|l|l|l|}
\hline Formulation & $\begin{array}{l}\text { Permeation Flux, Jss } \\
\left(\mathbf{\mu g} \mathbf{~ c m}^{-\mathbf{2}} \mathbf{h}^{-\mathbf{1}}\right)\end{array}$ & $\begin{array}{l}\text { Permeability } \\
\text { coefficient, Kp (cm } \\
\left.\mathbf{h}^{-\mathbf{1}}\right)\end{array}$ & $\begin{array}{l}\text { Enhancement } \\
\text { Ratio, Er }\end{array}$ & Skin retention (\%) \\
\hline $\begin{array}{l}\text { Vesicular } \\
\text { Dispersion }\end{array}$ & $7.34 \pm 0.61$ & 4.96 & 2.921 & $52.59 \pm 1.94 \%$ \\
\hline $\begin{array}{l}\text { Vesicular hydrogel } \\
\text { Conventional } \\
\text { hydrogel }\end{array}$ & $6.94 \pm 0.95$ & 4.68 & 2.682 & $54.45 \pm 3.09 \%$ \\
\hline
\end{tabular}

(Values are reported as mean \pm S.D; $n=3$ )

The amount of Resveratrol measured in the receptor compartments are the direct marker of transdermal delivery, while dermal delivery is indicated by the amount of Resveratrol retained in the skin layers. Thus, the extent of skin retention of Resveratrol through various developed hydrogels was evaluated. Resveratrol retained by vesicular dispersion, vesicular hydrogel, and traditional hydrogel in the dermal layer was quantified as $247.64 \pm 9.9 \mu \mathrm{g} \mathrm{cm}-2(52.59 \pm 1.94 \%), 254.85 \pm 5.27 \mu \mathrm{g}$ $\mathrm{cm}-2 \quad(54.45 \pm 3.09 \%)$, and $48.90 \pm 9.81 \mu \mathrm{g} \mathrm{cm}-2$ $(10.01 \pm 2.12 \%)$ respectively. The deposition of the skin by vesicular dispersion and the vesicular hydrogel is about five times greater than traditional hydrogel. The high deposition can be ascribed because vesicles in a deeper layer of skin are expected to form a depot. The present studies demonstrated the vesicular hydrogel's superior
Content of soya lecithin and Tween-80 in the oleic acid-based vesicles were found to be the major variables can be adequately controlled to achieve appropriate for topical use. As compared to conventional gel, permeation flux and skin deposition were also found to be improved. Thus, it can be concluded that Resveratrol loaded modified oleic acid vesicles could be an appropriate approach for topical delivery to treat UV-B induced precancerous skin lesions.

\section{Conflict of Interest}

The authors confirm that this article's content has no conflicts of interest.

\section{Acknowledgment}


The authors are thankful to the management of ISF College of Pharmacy (An autonomous Institute), Moga (Punjab), for providing the necessary facilities for the completion of this work

\section{References}

[1] Bragazzi NL, Sellami M, Salem I, et al. Fasting and its impact on skin anatomy, physiology, and physiopathology: A comprehensive review of the literature 2019; 11.

[2] Pasparakis M, Haase I, Nestle FO. Mechanisms regulating skin immunity and inflammation 2014; 14: 289-301.

[3] Wong R, Geyer S, Weninger W, et al. The dynamic anatomy and patterning of skin 2016; 25: 92-98.

[4] Losquadro WD. Anatomy of the Skin and the Pathogenesis of Nonmelanoma Skin Cancer 2017; 25: 283-89.

[5] Silpa SR, V C. a Review on Skin Cancer 2013; 4: 83-88.

[6] Liu-Smith F, Jia J, Zheng Y. UV-induced molecular signaling differences in melanoma and non-melanoma skin cancer 2017; 996: 27-40.

[7] Robinson K, Mock C, Liang D. Preformulation studies of resveratrol 2015; 41: $1464-$ 69.

[8] Elshaer $M$, Chen $Y$, Wang XJ, Tang X. Resveratrol: An overview of its anti-cancer mechanisms 2018; 207: 340-49.

[9] Sharma A, Sharma D, Baldi A, et al. Imiquimod-oleic acid prodrug loaded cream reduced drug crystallinity and induced indistinguishable cytotoxicity and apoptosis in mice melanoma tumour. vol. 2048. 2019.

[10] Zakir F, Vaidya B, Goyal AK, et al. Development and characterization of oleic acid vesicles for the topical delivery of fluconazole 2010; 17: 238-48.

[11] Kumar P, Singh S, Handa V, Kathuria H. Oleic Acid Nanovesicles of Minoxidil for Enhanced Follicular Delivery 2018; 5: 103.
[12] Negi P, Singh B, Sharma G, et al. Phospholipid microemulsion-based hydrogel for enhanced topical delivery of lidocaine and prilocaine: ObD-based development and evaluation 2016; 23: 951-67.

[13] Beg S, Saini S, Bandopadhyay S, Katare OP, et al. QbD-driven development and evaluation of nanostructured lipid carriers (NLCs) of Olmesartan medoxomil employing multivariate statistical techniques 2018; 44: 407-20.

[14] Garg BJ, Garg NK, Beg S, et al. Nanosized ethosomes-based hydrogel formulations of methoxsalen for enhanced topical delivery against vitiligo: Formulation optimization, in vitro evaluation and preclinical assessment 2016; 24: 233-46.

[15] Sharma G, Goyal H, Thakur K, et al. Novel elastic membrane vesicles (EMVs) and ethosomesmediated effective topical delivery of aceclofenac: a new therapeutic approach for pain and inflammation 2016; 23: 3135-45.

[16] Khurana B, Goyal AK, Budhiraja A, et al. Lipoplexes versus nanoparticles: PDNA/siRNA delivery 2013; 20: 57-64.

[17] Yadav NK, Nanda S, Sharma G, et al. Systematically optimized coenzyme q10-loaded novel proniosomal formulation for treatment of photo-induced aging in mice: Characterization, biocompatibility studies, biochemical estimations and anti-aging evaluation 2016; 24: 257-71. 